\title{
Pulmonary resection as treatment for haemorrhage in tuberculosis
}

\author{
E. V. LAUSTELA AND K. E. J. K YLLÖNEN \\ From the Clinic for Thoracic Surgery, Central Hospital, University of Helsinki
}

Pulmonary haemorrhage is a serious and not infrequent complication of tuberculosis. The cause of the haemorrhage is usually erosion of one of the branches of the pulmonary or bronchial artery in the walls of the cavity. Additional causes may be tuberculous bronchiectasis, cavernoliths or broncholiths. Until recently, the management of the pulmonary haemorrhage has been entirely conservative. The first published case of pulmonary resection for haemorrhage in tuberculosis was a pneumonectomy reported by Ryan and Lineberry in 1950. According to Rzepecki and Badmajew (1962), 54 cases of pulmonary haemorrhage, treated by resection, have been reported in the literature. Five of these ended fatally.

We report three cases of uncontrollable massive haemoptysis that were successfully treated by emergency pulmonary resection.

\section{CASE REPORTS}

CASE 1 The first patient was an electrician's wife aged 42 years. Pulmonary tuberculosis was discovered in 1947 , and two years later she was submitted to an extensive thoracoplasty in two phases because of a thick-walled cavity in the left upper lobe. There remained, however, a small residual cavity, and acidfast bacilli were occasionally cultivated from the sputa. She was given antituberculous drug therapy under ambulatory surveillance and was in good general condition. On 14 July 1959 she had an acute haemoptysis and was taken to the Laakso Hospital. The haemoglobin was then $9 \cdot 10 \mathrm{~g} . / 100 \mathrm{ml}$. (59\%). She was treated conservatively but did not improve in spite of frequent blood transfusions. Almost daily she had further attacks of haemorrhage, which were getting worse. On 3 September 1959 , more than $500 \mathrm{ml}$. of blood was coughed up. Since the situation was desperate and conservative treatment had failed, it was decided to operate. According to the radiographic examinations, the right lung had always been fairly sound, in contrast to the left lung which was, after the thoracoplasty, almost destroyed with a residual cavity and gross bronchiectasis. The left lung was considered to be the probable site of the haemorrhage.
On 4 September 1959 left pneumonectomy was per- $\stackrel{\vec{\omega}}{\vec{\omega}}$ formed under endotracheal anaesthesia with a selec- $\dot{\omega}$ tively occluding tube. For the operation the haemo- N globin was raised to $9.4 \mathrm{~g} . / 100 \mathrm{ml}$. $(61 \%)$ by rapid $\infty$ blood transfusions. The completely destroyed left lung 을 was extremely adherent to the chest wall, pericardium, and diaphragm. The site of the haemorrhage was in the dilated bronchi of the lower lobe, which were filled with blood clots. The patient recovered from the operation without any complications. There has been no haemoptysis since the operation. At the last routine examination in January 1963 she was in good condition with a low sedimentation rate and no acidfast bacilli in the sputa.

CASE 2 The second patient was an electrician aged 57 years, who in 1954 had an attack of haemoptysis. Radiographic examination of the chest revealed a shadow in the left upper lobe, and the sputum was positive for acid-fast bacilli. The patient was hospitalized for five months in Laakso Hospital, where he was given streptomycin, P.A.S., and isoniazid. In January 1956 he had another haemoptysis and was hospitalized for seven months. On admission, a cavity was found in the left upper lobe. During treatment the cavity decreased and the sputum was negative for acidfast bacilli when the patient left hospital.

On 17 May 1961 the patient was again admitted to Laakso Hospital because of haemoptysis, which had lasted for three days at home. Radiographic examinations revealed the same changes as before in the left upper field, with no new lesions. During two days in hospital the patient coughed up $2,600 \mathrm{ml}$. of blood and received eight pints of blood as transfusions to keep the blood pressure at the necessary level. As the pulmonary haemorrhage continued with the same violence, resection was proposed. Bronchoscopy, carried out in order to locate the site of the haemorrhage, revealed that the blood came from the left upper lobe. The patient was anaesthetized, and the right main bronchus was intubated. Left thoracotomy was done and the whole lung was found to be flooded with blood and inelastic. Finding the source of the bleeding by palpation was difficult. Because the radiographic examinations had shown that the lesion was situated in the apicoposterior segment, which moreover was the only part of the left lung adherent to the chest wall, this segment was removed. In the 
removed segment a thick-walled, blood-filled cavity and bronchiectasis were found. The histological picture corresponded to that of inactive pulmonary tuberculosis. Cultivation of the removed tissue did not reveal any acid-fast bacilli. After the operation the remaining left lung expanded completely and the patient recovered without complications. There has been no subsequent haemoptysis and the patient has been at work since March 1962.

CASE 3 The third patient was a chauffeur of 36 years. Pulmonary tuberculosis was detected in 1952, and he was treated in a sanatorium for six months. There was a cavity in the left upper lobe, but the patient refused surgical treatment and left the sanatorium. During the following years he had little trouble from his tuberculosis and only occasionally took antituberculous drugs. In the autumn of 1961 he had an episode of haematemesis and a radiographic examination showed a gastric ulcer. This was treated by vagotomy and gastro-enterostomy. On 24 October 1962 he had a sudden attack of copious haemoptysis and was admitted to Laakso Hospital. During the following days he had recurrent attacks of haemoptysis. The amounts of blood coughed up increased daily. On 1 November, he suddenly coughed up $1,800 \mathrm{ml}$. of blood and collapsed. He recovered from this attack in response to massive transfusions and oxygen inhalations, but his condition was very poor. He was cyanotic and had difficulty in breathing due to aspirated blood in the periphery of both lungs. To prevent a new and undoubtedly fatal attack of haemoptysis, operation was performed the next day. The site of the haemorrhage was considered to be the thick-walled cavity in the left upper lobe. At thoracotomy the left upper lobe was found to be extremely adherent to the chest wall and mediastinum. In the upper lobe several cavities could be palpated. Because the tuberculous changes involved the lower lobe, pneumonectomy was performed. Tracheostomy was done at the same time, and the patient was connected to a mechanical respirator. His condition continued to be critical for several days. After seven days the respirator was disconnected and the patient made a slow but smooth recovery.

In this case there were some diagnostic difficulties as to the origin of the haemorrhage: the patient had had a gastric ulcer with a history of haematemesis. In addition, during his attacks he also vomited blood. However, the site of the haemorrhage was considered to be the cavity in the left upper lobe. Post-operative examination showed a thick-walled cavity in the upper lobe, but the haemorrhage was caused by rupture of a large branch of the pulmonary artery into a wide bronchiectatic sac.

\section{DISCUSSION}

Until recently, pulmonary resection has not been considered useful in the management of haemorrhage in tuberculosis. In grave cases of massive haemoptysis, however, conservative treatment often fails. Cases in which failure of conservative treatment is imminent present an urgent indication for pulmonary resection. According to Rzepecki and Badmajew (1962), pulmonary resection has saved the patient in $91 \%$ of surgically treated cases. In our three cases the massive haemorrhage was effectively stopped by the resection, and the patients have had no further episodes of haemoptysis.

Accurate determination of the site of the haemorrhage is essential for successful surgical intervention. In our cases the tuberculous lesions were fortunately unilateral, and the site of the haemorrhage could be determined by pre-operative radiographic examinations and bronchoscopy. In bilateral cases the determination of the site of the haemorrhage may be difficult. Even in cases where the site of the haemorrhage is known, the contralateral lesions may present a contraindication to the operation by impairing the respiratory capacity. Rzepecki and Badmajew consider that contralateral lesions do not contraindicate an operation provided they are limited and inactive, or active but suitable for later surgical treatment.

During operation, placing the bleeding lesion by palpation may be difficult because of the massive post-haemorrhagic atelectasis of the lung, as in our second case. In such cases the pre-operative examinations must determine the site and extent of the resection.

According to Ross (1953), the essential conditions in which this type of operation should be performed include: (1) ideal anaesthesia ; (2) adequate replacement of blood loss ; (3) intrabronchial tamponade ; (4) early resection ; and (5) prolonged chemotherapy after the operation. In our cases points (1) and (3) were performed by selective occlusion of endobronchial tubes.

In these three cases resection was performed as an emergency operation in uncontrollable haemoptysis. Many tuberculous patients who have died because of massive pulmonary haemorrhage have had previous recurrent episodes of haemoptysis. Among these cases there may have been some patients who could have been saved by a timely operation. Conditions during a massive pulmonary haemorrhage are by no means optimal for a major operation because of the shock and the lowered respiratory efficiency. Consequently, in our opinion, haemorrhagic episodes in tuberculosis should be considered as strong indications for resection, even if the lesions per se do not form an absolute indication for surgical treatment. Lately 
we have operated on two cases in the silent phase ; the first one after an episode of controlled massive haemoptysis, the other after prolonged haemoptysis.

\section{SUMMARY}

The authors report three cases of uncontrollable massive pulmonary haemorrhage in tuberculosis which were successfully treated by resection. They suggest that recurrent haemoptyses should be con- $\overrightarrow{\bar{C}}$ sidered an indication for pulmonary resection.

\section{REFERENCES}

Ross, C. A. (1953). Emergency pulmonary resection for massive hemoptysis in tuberculosis. J. thorac. Surg., 26, 435.

Ryan, T. C., and Lineberry, W. T., Jr. (1950). Pneumonectomy for pulmonary hemorrhage in tuberculosis. Amer. Rev. Tuberc., 61, 426.

Rzepecki, W., and Badmajew, P. (1962). Hemorrhage in tuberculosis as an urgent indication for resection of pulmonary tissue. Dis. Chest, 41, 372. 